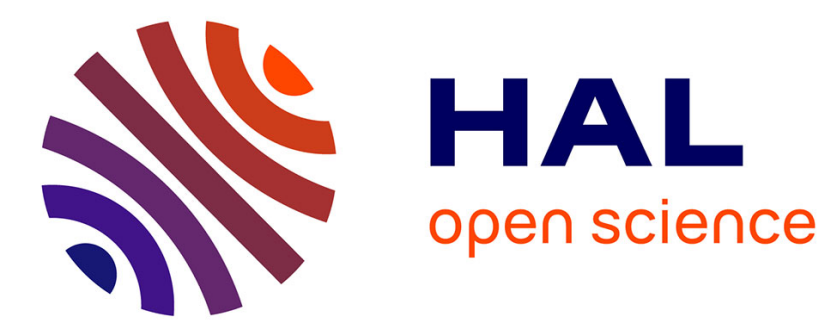

\title{
Performance of DSTM MIMO Systems in Continuously Changing Rayleigh Channel
}

\author{
Hui Ji, Gheorghe Zaharia, Jean-François Hélard
}

\section{To cite this version:}

Hui Ji, Gheorghe Zaharia, Jean-François Hélard. Performance of DSTM MIMO Systems in Continuously Changing Rayleigh Channel. International Symposium of Signals, Circuits \& Systems (ISSCS 2015), Jul 2015, Iasi, Romania. 10.1109/isscs.2015.7203999 . hal-01175631

\section{HAL Id: hal-01175631 \\ https://hal.science/hal-01175631}

Submitted on 10 Jul 2015

HAL is a multi-disciplinary open access archive for the deposit and dissemination of scientific research documents, whether they are published or not. The documents may come from teaching and research institutions in France or abroad, or from public or private research centers.
L'archive ouverte pluridisciplinaire HAL, est destinée au dépôt et à la diffusion de documents scientifiques de niveau recherche, publiés ou non, émanant des établissements d'enseignement et de recherche français ou étrangers, des laboratoires publics ou privés. 


\title{
Performance of DSTM MIMO Systems in Continuously Changing Rayleigh Channel
}

\author{
Hui Ji, Gheorghe Zaharia, Jean-François Hélard \\ Université Européenne de Bretagne (UEB) \\ INSA, IETR, UMR 6164, F-35708, Rennes, France \\ Email:\{hui.ji; gheorghe.zaharia; jean-francois.helard\}@insa-rennes.fr
}

\begin{abstract}
It is well proved that the signals transmitted through a mutipath channel experience Rayleigh fading. The channel also varies due to the moving of user end or the scatters and Doppler shift effect is generated. Researchers have to consider these two effects in their studies of wireless communications. Some researchers use step channel model which is too idealized to simulate MIMO systems. Besides, sum-of-sinusoid based simulators are widely used among which Jakes' simulator is the most well-known. However this kind of simulator has to strive for the deficiencies in higher-order statistics due to the approximation of random variables by determinants. In this paper, we propose a time selective channel model used for laboratory simulations from a new sight which can avoid the above drawbacks. The channel is reconstructed by sinc function based on Nyquist's sampling theorem. The performance of DSTM scheme is evaluated in this new channel model.
\end{abstract}

Keywords - time-selective channel model, Rayleigh fading, sinc interpolation, Nyquist's sampling theorem, DSTM.

\section{INTRODUCTION}

In the study of modern mobile or wireless communication systems, people have to combat Rayleigh fading due to the multipath transmission. Doppler effect is another problem we have to overcome. In laboratory researches, appropriate modelization of channel with these two characters is important.

In our study of designing MIMO systems without channel state information, we find that researchers mainly use two kinds of channel models: step channel model and Jakes' model. Tarokh and Jafarkhani used the step channel model when presented the DSTBC (differential space-time block coding) scheme in [1], [2]. Hughes also employed this channel model in [3] when he proposed the DSTM (differential spacetime modulation). The multipath channel is assumed to be quasi-static so that the channel coefficients are constant over a frame of length $L$ and vary from one frame to another. We believe that this model is too idealized. Besides, Jakes' model is widely used by many researchers. In fact Jakes' simulator is derived from Clarke's mathematical model where the fading is time-correlated. Hochwalds and Swelden used Jakes' simulator when they presented DUSTM (differential unitary space-time modulation) in [4]. However, Jakes' simulator is non-stationary [5]. Other improved sum-of-sinusoid based simulators are proposed in [5]-[7]. The sum-of-sinusoid simulator is just an approximation of Clarke's mathematical model which leads some degrees of loss of the statistical properties such as deficiencies in higher-order statistics.
Here, we propose a time-selective Rayleigh fading channel model based on the assumption that the changing rate of channel is limited to $f_{d}$ (the maximum Doppler frequency). The channel can be reconstructed by the samples obtained with frequency greater than $2 f_{d}$ with the sinc interpolation function. This model is originally presented in [8]. However the channel is block-constant, i.e., the channel is assumed to be constant during the transmission of a symbol matrix. In this paper, we expand the idea to a continuously changing channel. The channel changes slightly for successive transmitted symbols, i.e. each column of a transmitted matrix is computed using a different channel matrix. The performance of DSTM schemes under this new channel is investigated.

\section{Models OF SISO RAdio CHANNEL}

\section{A. Clarke's channel model}

The mathematical channel model widely used today is established by Clarke in his fundamental work [9]. The baseband complex impulse response of SISO radio channel is:

$$
h(t)=\sum_{n=1}^{N_{p}} \alpha_{n} \exp \left[j\left(2 \pi f_{d} t \cos \theta_{n}+\phi_{n}\right)\right],
$$

where $N_{p}$ is the number of propagation paths, $0<\alpha_{n}<1$ is the attenuation of the $n^{\text {th }}$ path, $f_{d}$ is the maximum Doppler frequency and $\theta_{n}$ and $\phi_{n}$ are, respectively, the angle of arrival and random phase of the $n^{t h}$ propagation path. Both $\theta_{n}$ and $\phi_{n}$ are uniformly distributed over $[-\pi, \pi)$ for all $n$ and they are mutually independent.

The autocorrelation of $h(t)$ is given by [10]:

$$
R_{h}(\tau)=\mathbb{E}[h(t) h(t+\tau)]=2 \sigma^{2} J_{0}\left(2 \pi f_{d} \tau\right),
$$

where $\sigma^{2}=0.5 \sum_{n} \mathbb{E}\left[\alpha_{n}^{2}\right]$ and $J_{0}(\cdot)$ is the zero-order Bessel function of the first kind. Conventionally, people assume that $\sum_{n} \mathbb{E}\left[\alpha_{n}^{2}\right]=1$ to ensure that the received signal power is equal to the transmitted signal power which results in $R_{h}(\tau)=$ $J_{0}\left(2 \pi f_{d} \tau\right)$.

The envelope of the received signal $z(t)=|h(t)|$ is Rayleigh-distributed. The maximum Doppler shift is defined as $f_{d}=V / \lambda$, where $V$ is the relative velocity between the transmitter and receiver, and $\lambda$ is the signal wavelength. During the coherence time $T_{c}$, the channel characteristics do not change significantly. Clearly, a slow-changing channel has a large coherence time. There is no exact relationship between 
coherence time and Doppler spread. A popular definition of $T_{c}$ is: $T_{c}=\sqrt{\frac{9}{16 \pi f_{d}^{2}}}$ [11]. In practice, for simplicity, people usually use it as $T_{c} \approx 0.5 / f_{d}$.

In [4], [12], the authors use Jakes' model [13] which approximates Clarke's model by setting equal strength multipath components, i.e., $\alpha_{n}=\frac{1}{\sqrt{N}}$ and choosing the $N$ angles $\theta_{n}$ to be uniformly distributed.

\section{B. The step channel model}

The channel model used in [1], [3], [14] is constant during one frame and changes randomly for the next frame. Define $L=T_{c} / T_{s}$, where $T_{s}$ is the symbol duration time, the channel is assumed to be constant during the transmission of $L$ symbols. For example, with $f_{d}=100 \mathrm{~Hz}, T_{c}=5$ $\mathrm{ms}$ and $T_{s}=25 \mu \mathrm{s}$, the normalized coherence interval is $L=200$, during the transmission of the first frame of 200 symbols, the same channel coefficients matrix is used. The next channel coefficients matrix is randomly generated to be used for the next 200 symbols. When analyzing the performances of DSTM MIMO systems using the step model, due to the discontinuousness between two successive frames, at the beginning of each new frame, the reference signal matrix has to be transmitted.

\section{The New Improved Channel Model}

Using the well-known Nyquist's sampling theorem, a bandlimited signal $x(t)$ can be reconstructed from its samples $x\left(k T_{0}\right)$ with sinc function if the sampling frequency $f_{0}=$ $1 / T_{0}>2 f_{M}$, where $f_{M}$ is the maximum frequency of the signal. We reconstruct the channel based on this idea.

The channel $h(t)$ can be seen as a complex Gaussian random process. We know that the function $J_{0}(x)$ has its first zero-point at $x \approx 2.4048$. It is reasonable to suppose that the channel coefficients separated by $\tau_{0}=2.4048 /\left(2 \pi f_{d}\right) \approx$ $0.3827 / f_{d}$ are uncorrelated. If the random variables obtained by sampling a Gaussian process $h(t)$ are uncorrelated, these random variables are statistically independent. It is clear that the function $h(t)$ in (1) has the maximum frequency $f_{d}$. If we try to reconstruct $h(t)$, the sampling frequency should be $f_{0}>2 f_{d}$ and the sample period $T_{0}<0.5 / f_{d}$. Notice that $\tau_{0}<0.5 / f_{d}$, therefore it is possible to reconstruct channel response with independently generated Rayleigh distributed random variables.

The channel $h(t)$ can be reconstructed by random variables $r_{k}=g_{k r}+j g_{k i}, \quad k=1,2, \ldots, K$, where $g_{k r}$ and $g_{k i}$ are Gaussian distributed random variables with mean zero and variance 0.5 . In this case the envelope of $r_{k}$ is Rayleigh distributed. We assume that $r_{k}(k=1,2, \ldots, K)$ are separated by $\tau_{0}=2.4048 /\left(2 \pi f_{d}\right) \approx 0.3827 / f_{d}$. With these $K$ randomly generated points, $h(t)$ can be constructed by

$$
h(t)=\sum_{k=0}^{K-1} r_{k} \frac{\sin \pi\left(f_{0} t-k\right)}{\pi\left(f_{0} t-k\right)},
$$

where $T_{0}=\tau_{0}=2.4048 /\left(2 \pi f_{d}\right) \approx 0.3827 / f_{d}$ and $f_{0}=$ $1 / T_{0} \approx f_{d} / 0.3827$. In [8], we propose a block-constant

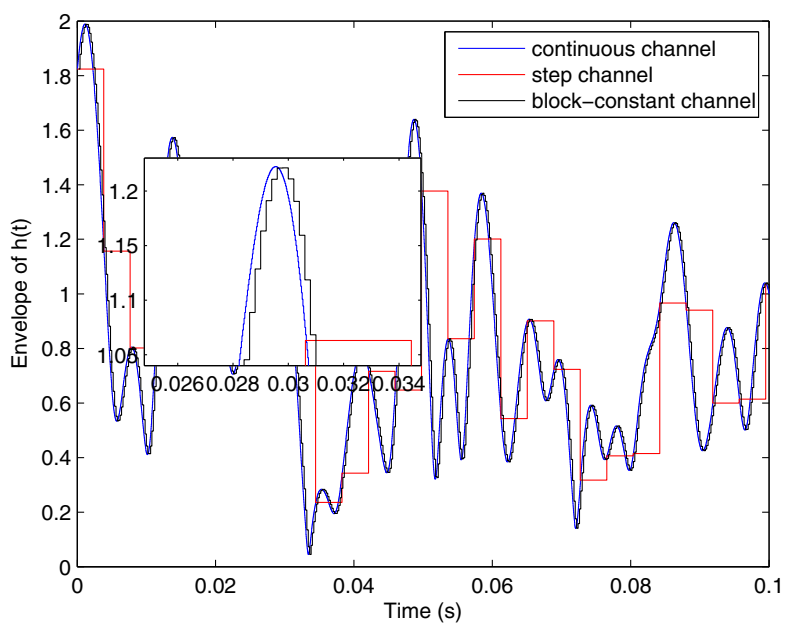

Fig. 1. Channel interpolation with $f_{d}=100 \mathrm{~Hz}, T_{s}=25 \mu \mathrm{s}$.

Rayleigh channel model for DSTM schemes. For MIMO systems with $M$ transmit antennas, the channel is supposed to be constant during the transmission of one matrix with $T=M$ symbols. However, two successive independently generated random variables are supposed to be separated by $T_{0}=T_{c}$, while in Clarke's mathematical channel model, the channel coefficients separated by $T_{c}$ have correlation $J_{0}\left(2 \pi f_{d} T_{c}\right)=J_{0}(\pi) \approx-0.3$. With this model, we should interpolate $N_{m}-1=L / T-1$ coefficients between two random variables. The channel coefficient used for the $i_{t h}$ transmission matrix is

$$
h(i)=\sum_{k=0}^{K-1} r_{k} \frac{\sin \pi\left[f_{0}\left(i M T_{s}\right)-k\right]}{\pi\left[f_{0}\left(i M T_{s}\right)-k\right]} .
$$

In this paper, the channel coefficient used for the $i_{t h}$ symbol is

$$
h(i)=\sum_{k=0}^{K-1} r_{k} \frac{\sin \pi\left[f_{0}\left(i T_{s}\right)-k\right]}{\pi\left[f_{0}\left(i T_{s}\right)-k\right]} .
$$

We compare the step channel, the block-constant channel and the continuous channel in Fig. 1. The number of transmit antennas is $M=8$, the maximum Doppler frequency $f_{d}=$ $100 \mathrm{~Hz}, T_{s}=25 \mu \mathrm{s}$ and $L=T_{c} / T_{s}=200$.

\section{Performance of DSTM in Continuously Changing RAYleigh CHANNEL}

\section{A. The DSTM MIMO system}

Generally, a MIMO system with $M$ transmit antennas and $N$ receive antennas can be written as:

$$
\mathbf{y}_{t}=H_{t} \mathbf{x}_{t}+\mathbf{w}_{t}
$$

where $t$ is the time index, $\mathbf{x}_{t}$ is the $M \times 1$ transmission symbol vector. $H_{t}$ is the $M \times N$ channel matrix at time $t$. $\mathbf{w}_{t}$ is the $N \times 1$ complex, additive white Gaussian noise vector and $\mathbf{y}_{t}$ is the $N \times 1$ received symbol vector. 
DSTM schemes are normally designed with the assumption that the channel changes slightly during the coherence time $T_{c}$ and is constant during the transmission of two successive matrices of symbols. The matrix form of MIMO systems is $Y=H X+W$, where $X$ is the $M \times T$ transmission matrix and $T$ denotes the number of symbols transmitted by each antenna. $W$ is the $N \times T$ Gaussian noise matrix and $Y$ is the $N \times T$ matrix of the received signals.

For differential space-time modulation systems, the information matrix is used to multiply the previous transmitted matrix. In general, the information matrix is selected from a group $P$ according to the incoming information bits. For example, at time $\tau, X_{\tau}$ is transmitted. At the next time $\tau+1$, a block of information bits is mapped onto the matrix $V_{i_{\tau+1}}$ from the group $P$, and then the matrix

$$
X_{\tau+1}=X_{\tau} V_{i_{\tau+1}}
$$

is transmitted. All the matrices of the group $P$ are unitary matrices. At the very beginning, a reference matrix $V_{0}$ is transmitted, i.e., $X_{0}=V_{0}$.

At the receiver end, a matrix stream $Y_{0}, \ldots, Y_{\tau}, Y_{\tau+1}, \ldots$ is detected by the receive antennas, i.e.,

$$
\begin{aligned}
Y_{\tau} & =H_{\tau} X_{\tau}+W_{\tau} \\
Y_{\tau+1} & =H_{\tau+1} X_{\tau+1}+W_{\tau+1}
\end{aligned}
$$

With the assumption that the channel is constant during the transmission of $X_{\tau}$ and $X_{\tau+1}$, using the differential transmission equation (7), we get

$$
Y_{\tau+1}=Y_{\tau} V_{i_{\tau+1}}+W_{\tau+1}^{\prime}
$$

where $W_{\tau+1}^{\prime}=W_{\tau+1}-W_{\tau} V_{i_{\tau+1}}$.

As $Y_{\tau}$ and $Y_{\tau+1}$ are known by the receiver, we can use the maximum likelihood detector to estimate the information matrix:

$$
\hat{V}_{i_{\tau+1}}=\arg \min _{V_{l} \in P}\left\|Y_{\tau+1}-Y_{\tau} V_{l}\right\|
$$

In [8], the performance degradation of the DSTM MIMO systems with block-constant channel compared to their performance obtained with step channel is analysed. Here, we suppose the channel changes continuously and evaluate the performance of DSTM schemes in this channel.

\section{B. The constellation of the DSTM schemes}

In our scheme, the transmitted matrices are based on the Weyl group used in [15]. The Weyl group $G_{w}$ is a set that contains 12 cosets $C_{0}, C_{1}, \ldots, C_{11}$. Each coset contains 16 invertible matrices. The first coset is defined as:

$$
C_{0}=\alpha\left\{\left[\begin{array}{ll}
1 & 0 \\
0 & 1
\end{array}\right],\left[\begin{array}{cc}
1 & 0 \\
0 & -1
\end{array}\right],\left[\begin{array}{ll}
0 & 1 \\
1 & 0
\end{array}\right],\left[\begin{array}{cc}
0 & 1 \\
-1 & 0
\end{array}\right]\right\}
$$

with $\alpha \in\{1,-1, i,-i\}$. The 12 cosets of $G_{w}$ are derived from $C_{0}$ as follows:

$$
C_{k}=A_{k} C_{0}, \forall k=0,1, \ldots, 11,
$$

where the matrices $A_{k}, k=0,1, \ldots, 5$ are respectively:

$$
\begin{array}{r}
A_{0}=\left[\begin{array}{ll}
1 & 0 \\
0 & 1
\end{array}\right], A_{1}=\left[\begin{array}{ll}
1 & 0 \\
0 & i
\end{array}\right], A_{2}=\frac{1}{\sqrt{2}}\left[\begin{array}{cc}
1 & 1 \\
1 & -1
\end{array}\right], \\
A_{3}=\frac{1}{\sqrt{2}}\left[\begin{array}{cc}
1 & 1 \\
i & -i
\end{array}\right], A_{4}=\frac{1}{\sqrt{2}}\left[\begin{array}{cc}
1 & i \\
1 & -i
\end{array}\right], A_{5}=\frac{1}{\sqrt{2}}\left[\begin{array}{ll}
1 & i \\
i & 1
\end{array}\right],
\end{array}
$$

and the matrices $A_{k}, k=6,7, \ldots, 11$ are given by:

$$
A_{k+6}=\eta A_{k}, \text { with } \eta=(1+i) / \sqrt{2}, \forall k=0,1, \ldots, 5
$$

1) DSTM scheme with 2 transmit antennas: In this paper, for MIMO systems with 2 transmit antennas and $R=1$ bps/Hz, 4 matrices are needed. We select matrices $\left[\begin{array}{ll}1 & 0 \\ 0 & 1\end{array}\right]$, $\left[\begin{array}{cc}1 & 0 \\ 0 & -1\end{array}\right],\left[\begin{array}{ll}0 & 1 \\ 1 & 0\end{array}\right]$, and $\left[\begin{array}{cc}0 & 1 \\ -1 & 0\end{array}\right]$ as the information set. For $R=2$ bps/Hz, we select $C_{0}$ which has 16 matrices as the group to map the 4 bits information block as in [14].

2) DSTM scheme with 4 transmit antennas: For MIMO systems with 4 transmit antennas, the Kronecker product is used to expand the $2 \times 2$ Weyl group to a $4 \times 4$ matrices group. For $R=1 \mathrm{bps} / \mathrm{Hz}$, the first matrix in $C_{0}\left(\left[\begin{array}{ll}1 & 0 \\ 0 & 1\end{array}\right]\right)$ is used to make Kronecker product with all the matrices in $C_{0}$ to get 16 unitary matrices as in [16].

For $R=2 \mathrm{bps} / \mathrm{Hz}$, the best set used in [16] which contains 256 matrices is used here. In fact, the first 16 matrices from every successive 192 matrices of the group $G_{w 4}$ are selected to form the set.

3) DSTM scheme with 8 transmit antennas: For MIMO systems with 8 transmit antennas and $R=1 \mathrm{bps} / \mathrm{Hz}, 256$ matrices should be generated as the mapping group. We get the group as follows. First, we generate a set of 16 matrices of $C_{44}$ by using the Kronecker product between the first 4 matrices of $C_{0}$. Second, the Kronecker product between $C_{0}$ (16 matrices of the size $2 \times 2$ ) and $C_{44}$ (16 matrices of the size $4 \times 4$ ) produces a set $C_{88}$ with 256 matrices.

\section{Error performance of DSTM with new channel model}

The performance of the differential MIMO systems are evaluated over the frame constant channel (step channel), the block-constant channel and the continuous channel. We set $L=200$, i.e., $T_{c} / T_{s}=200$, that means for $f_{d}=100 \mathrm{~Hz}$, $T_{s}=25 \mu \mathrm{s}$ and symbol rate $f_{s}=40 \mathrm{kHz}$.

Fig. 2 shows that for $R=1 \mathrm{bps} / \mathrm{Hz}$, with the normalize de coherence time $L=200$, DSTM schemes over continuous channel perform similar to those over step channel. However, DSTM schemes perform better than those over block-constant channel, which is resulted from the less value of discontinuity of the channel coefficients for two successively transmitted symbols compared to step channel. Similar relative results for $R=2 \mathrm{bps} / \mathrm{Hz}, \mathrm{M} 8 \mathrm{~N} 8, \mathrm{M} 4 \mathrm{~N} 4$ and M2N2 schemes are obtained in Fig. 3.

Fig. 4 presents the performance of M4N1 DSTM scheme with $R=1 \mathrm{bps} / \mathrm{Hz}$ over the step channel and the continuous channel with different normalized coherence time L. The simulation results show that the smaller the coherence time interval is, which means the fading rate is high, the worse the BER performance will be. 


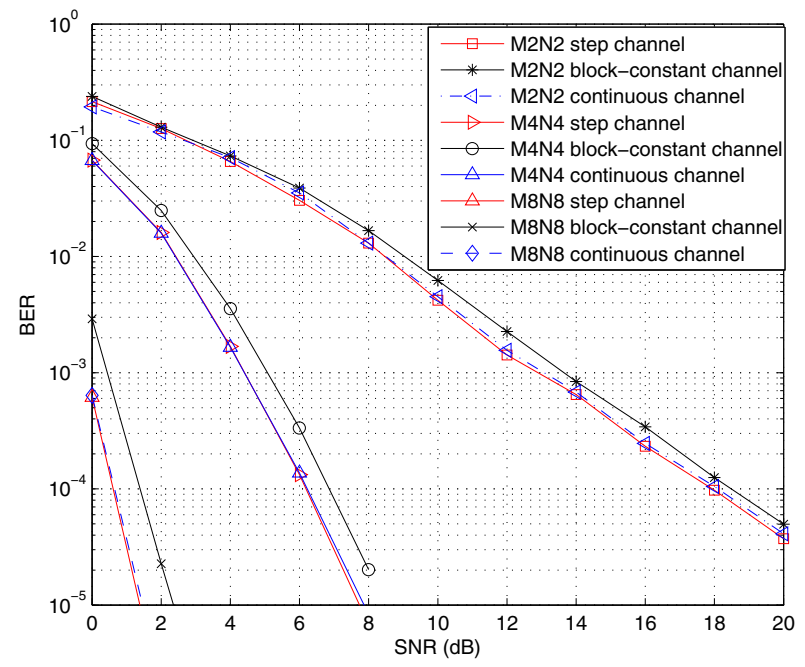

Fig. 2. Performances of differential space-time schemes with $R=1 \mathrm{bps} / \mathrm{Hz}$ over different channel models.

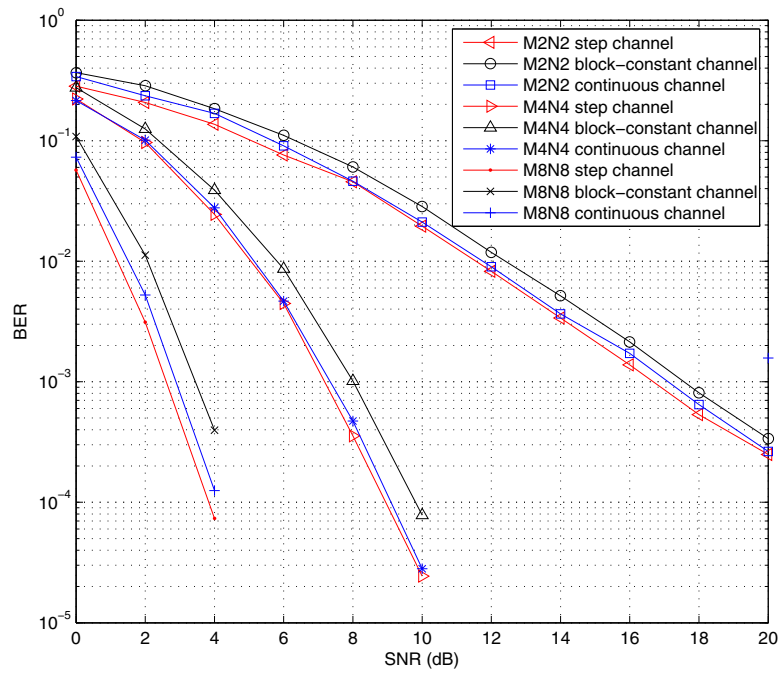

Fig. 3. Performances of differential space-time schemes with $R=2 \mathrm{bps} / \mathrm{Hz}$ over different channel models.

\section{CONCLUSION}

In this paper, we propose a new continuous time-selective channel model for differential space-time modulation schemes. This channel model is more realistic that the step channel which is constant during a fixed time and more precise than the sum-of-sinusoid channel models. The channel is reconstructed based on Nyquist's sampling theorem and obtained by interpolation of sinc functions. We evaluate the performances of DSTM schemes with 2, 4 and 8 transmit antennas in these channels. Simulation results show that over continuous channel, DSTM schemes perform better than those over blockconstant channel, which is resulted from the less value of discontinuity of the channel coefficients for two successively

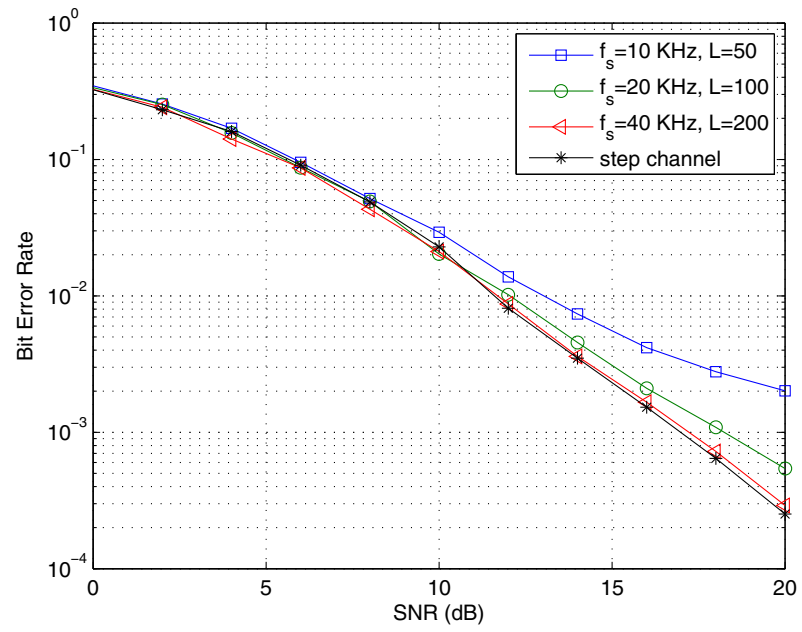

Fig. 4. Performance of the DSTM M4N4R1 scheme with different $L$.

transmitted symbols.

\section{REFERENCES}

[1] V. Tarokh and H. Jafarkhani, "A differential detection scheme for transmit diversity," IEEE J. Select. Areas Commun., vol. 18, no. 7, pp. 1169-1174, 2000

[2] H. Jafarkhani and V. Tarokh, "Multiple transmit antenna differential detection from generalized orthogonal designs," IEEE Trans. Inform. Theory, vol. 47, no. 6, pp. 2626-2631, 2001.

[3] B. L. Hughes, "Differential space-time modulation," IEEE Trans. Inform. Theory, vol. 16, no. 7, pp. 2567-2578, 2000.

[4] B. M. Hochwald and W. Sweldens, "Differential unitary space time modulation," IEEE Trans. Commun., vol. 48, no. 12, pp. 2041-2052, 2000.

[5] M. F. Pop and N. C. Beaulieu, "Limitations of sum-of-sinusoids fading channel simulators," IEEE Trans. Commun., vol. 49, pp. 699-708, April 2001.

[6] Y. R. Zheng and C. Xiao, "Simulation models with correct statistical properties for Rayleigh fading channels," IEEE Trans. Commun., vol. 51, pp. 920-928, June 2003.

[7] C. Xiao, Y. R. Zheng, and N. C. Beaulieu, "Novel sum-of-sinusoids simulation models for Rayleigh and Rician fading channels," IEEE Trans. Wireless Commun., vol. 5, pp. 3667-3679, December 2006.

[8] H. Ji, G. Zaharia, and J.-F. Hélard, "Performance of DSTM MIMO systems based on Weyl group in time selective channel," in European Wireless, 2014.

[9] R. H. Clarke, "A statistical theory of mobile-radio reception," Bell Labs Tech. J., pp. 957-1000, Jul.-Aug. 1968.

[10] G. L. Stüber, Principles of Mobile Communication. Kluwer Academic Publlishers, 2nd ed., 2001.

[11] B. Sklar, Rayleigh Fading Channels, The mobile communications handbook. CRC Press, 1999.

[12] B. M. Hochwald and T. L. Marzetta, "Unitary space-time modulation for multiple-antenna communications in Rayleigh flat fading," IEEE Trans. Inform. Theory, vol. 46, no. 2, pp. 543-564, 2000.

[13] W. C. Jakes and D. C. Cox, Microwave mobile communications. WileyIEEE Press, 1994

[14] H. Ji, G. Zaharia, and J.-F. Hélard, "A new differential space-time modulation scheme for MIMO systems with four transmit antennas," in 20th International Conference on Telecommunications (ICT), 2013.

[15] A. El Arab, J.-C. Carlach, and M. Hélard, "A new non-coherent MIMO scheme: Matrix coded modulation 'MCM',' in Internat. Symp. on Commun. and Inform. Technal. (ISCIT), pp. 120-125, 2011.

[16] H. Ji, G. Zaharia, and J.-F. Hélard, "A new differential space-time modulation scheme based on weyl group," in the 11-th International Symposium on Signals, Circuits and Systems (ISSCS), 2013. 